\title{
Heavy-Ion Collisions at LHC in a Multiphase Transport Model
}

\author{
Che Ming $\mathrm{Ko}^{1}$, Lie-Wen Chen ${ }^{2,3}$, and Ben-Wei Zhang ${ }^{1} *$ \\ ${ }^{1}$ Cyclotron Institute and Physics Department, Texas A\&M University, College Station, Texas 77843-3366 USA \\ ${ }^{2}$ Institute of Theoretical Physics, Shanghai Jiao Tong University, Shanghai 200240, China and \\ ${ }^{3}$ Center of Theoretical Nuclear Physics, National Laboratory of Heavy Ion Accelerator, Lanzhou 730000, China
}

\section{Received on 28 December, 2006}

\begin{abstract}
Using a multiphase transport (AMPT) model that includes scatterings in both initial partonic and final hadronic matters as well as the transition between these two phases of matter, we make predictions on the rapidity distributions and transverse momentum spectra of various hadrons, their elliptic flows, and two-pion correlation functions in ultra-relativistic heavy ion collisions at the Large Hadron Collider (LHC).
\end{abstract}

Keywords: Heavy-ion collisions; LHC; Transport model; Rapidity distributions; Transverse momentum spectra; Elliptic flow; Pion interferometry

\section{INTRODUCTION}

Many observables have been proposed as possible signatures for the deconfined plasma of quarks and gluons that is expected to be produced during the initial stage of ultrarelativistic heavy ion collisions. These include enhanced production of dileptons of intermediate invariant masses [1] and baryons made of multi-strange quarks [2], increased emission duration [3, 4], suppressed production of charmonia [5], large anisotropic flows of hadrons [6], quenching of minijets with large transverse momenta [7], and scaling of hadron elliptic flows according to their constituent quark content [8]. Most of these observables have been studied during past few years in experiments at the Relativistic Heavy Ion Collider (RHIC) involving $\mathrm{Au}+\mathrm{Au}$ collisions at center-of-mass energies $\sqrt{s_{N N}}=130$ and $200 \mathrm{GeV}$. Studying these signatures using various theoretical models, such as the statistical model $[9,10]$, the hydrodynamic model [11-13], the transport model [14-23], the quark coalescence model [24-27], and the perturbative QCD approach [28, 29], has provided convincing evidence that the quark-gluon plasma has indeed been produced in these collisions. Moreover, these studies have indicated that the quark-gluon plasma produced at RHIC is strongly interacting with transport coefficients much larger than those given by the perturbative QCD [30].

Since heavy ion collisions at energies much higher than that at RHIC will soon be available at the Large Hadron Collider (LHC), it is of interest to make predictions for above mentioned observables based on what we have learnt from heavy ion collisions at RHIC. Using a multiphase transport (AMPT) model, which has been quite useful in understanding experimental results at RHIC, we have carried out such a study. In particular, we have made predictions on the rapidity distributions and transverse momentum spectra of various hadrons, their elliptic flows, and two-pion correlation functions in $\mathrm{Pb}+\mathrm{Pb}$ collisions at center-of-mass energy of $\sqrt{s_{N N}}=5.5 \mathrm{TeV}$ that is to be available at LHC.

* On leave from Institute of Particle Physics, Huazhong Normal University, Wuhan 430079, China

\section{THE AMPT MODEL}

Before presenting predicted results, we review briefly the AMPT model [14-16, 31-34]. It is a hybrid model that uses minijet partons from hard processes and strings from soft processes in the heavy ion jet interaction generator (HIJING) model [35] as initial conditions for modelling heavy-ion collisions at ultra-relativistic energies. Time evolution of resulting minijet partons, which are largely gluons, is described by Zhang's parton cascade (ZPC) model [36]. At present, this model includes only parton-parton elastic scatterings with an in-medium cross section given by the perturbative QCD, i.e.,

$$
\frac{d \sigma_{p}}{d t} \simeq \frac{9 \pi \alpha_{s}^{2}}{2\left(t-\mu^{2}\right)^{2}}, \quad \sigma_{p} \simeq \frac{9 \pi \alpha_{s}^{2}}{2 \mu^{2}} \frac{1}{1+\mu^{2} / s},
$$

where $\alpha_{s}$ is the strong coupling constant and is taken to have a value of 0.47 , and $s$ and $t$ are the usual Mandelstam variables for squared center-of-mass energy and four momentum transfer, respectively. The effective screening mass $\mu$ depends on the temperature and density of partonic matter but is taken as a parameter in ZPC for fixing the magnitude and angular distribution of parton scattering cross section. After minijet partons stop interacting, they are combined with their parent strings, as in the HIJING model with jet quenching, to fragment into hadrons using the Lund string fragmentation model as implemented in the PYTHIA program [37]. The final-state hadronic scatterings are modelled by a relativistic transport (ART) model $[38,39]$. This default AMPT model has been quite successful in describing measured rapidity distributions of charged particles, particle to antiparticle ratios, and spectra of low transverse momentum pions and kaons in heavy ion collisions at the Super Proton Synchrotron (SPS) and RHIC [15]. It has also been useful in understanding the production of $J / \psi[31]$ and multistrange baryons [34] in these collisions.

Since the initial energy density in ultra-relativistic heavy ion collisions is expected to be much larger than the critical energy density at which the hadronic matter to quark-gluon plasma transition would occur $[16,31,40]$, the AMPT model has been extended to allow initial excited strings to melt into partons [41]. In this version, hadrons that would have been produced from the HIJING model are converted to their valence quarks and/or antiquarks. Interactions among these par- 
tons are again described by the ZPC parton cascade model. Because inelastic scatterings are not included in the current version of the ZPC model, only quarks and antiquarks from melted strings are present in the partonic matter. The species independence of the cross section used in the ZPC model compensates, however, for the absence of gluons in the early stage.

The transition from the partonic matter to the hadronic matter in the AMPT with string melting is achieved using a coordinate-space quark coalescence model, i.e., two nearest quark and antiquark are combined into mesons and three nearest quarks or antiquarks are combined into baryons or antibaryons that are closest to the invariant masses of these parton combinations. This coalescence model is somewhat different from the ones that are based on the overlap of hadron quark wave functions with the quark distribution functions in the partonic matter and used extensively for studying the production of hadrons with intermediate transverse momenta [24-26]. The final-state scatterings of produced hadrons from quark coalescence are again described by the ART model.

Using parton scattering cross sections of $6-10 \mathrm{mb}$, the AMPT model with string melting is able to reproduce the centrality and transverse momentum (below $2 \mathrm{GeV} / c$ ) dependence of hadron elliptic flows [41] and higher-order anisotropic flows [42] as well as the pion interferometry [43] measured in $\mathrm{Au}+\mathrm{Au}$ collisions at $\sqrt{s_{N N}}=130 \mathrm{GeV}$ at RHIC [44-46]. It has also been used for studying the kaon interferometry [47] in these collisions as well as many other observables at $\sqrt{s_{N N}}=200 \mathrm{GeV}$, such as the pseudorapidity [48], system size [49, 50], and flavor [51, 52] dependence of anisotropic flows.

\section{RESULTS}

Using the AMPT model, we have studied $\mathrm{Pb}+\mathrm{Pb}$ collisions at center-of-mass energy of $\sqrt{s_{N N}}=5.5 \mathrm{TeV}$ to be available at LHC, which is an order of magnitude higher than that at RHIC. Observables shown in the following include the rapidity distributions and transverse momentum spectra of various hadrons, their elliptic flows, and two-pion correlation functions.

\section{A. Rapidity distributions}

The number of particles produced in a heavy ion collision provides valuable information on the energy density of the matter formed during the initial stage. Since the multiplicity of produced particles in the AMPT model is essentially determined by the initial conditions taken from the HIJING model through the hard and soft processes among colliding nucleons, the default AMPT model is sufficient for this study. Shown in Fig. 1 are the charged particle pseudorapidity distribution (upper left panel) and the rapidity distributions of charged pions (lower left panel), kaons (lower right panel) as well as protons and antiprotons (upper right panel) in central $(b \leq 3 \mathrm{fm}) \mathrm{Pb}+\mathrm{Pb}$ collisions at $\sqrt{s_{N N}}=5.5 \mathrm{TeV}$. Both results obtained with (lines with circles) and without (lines without
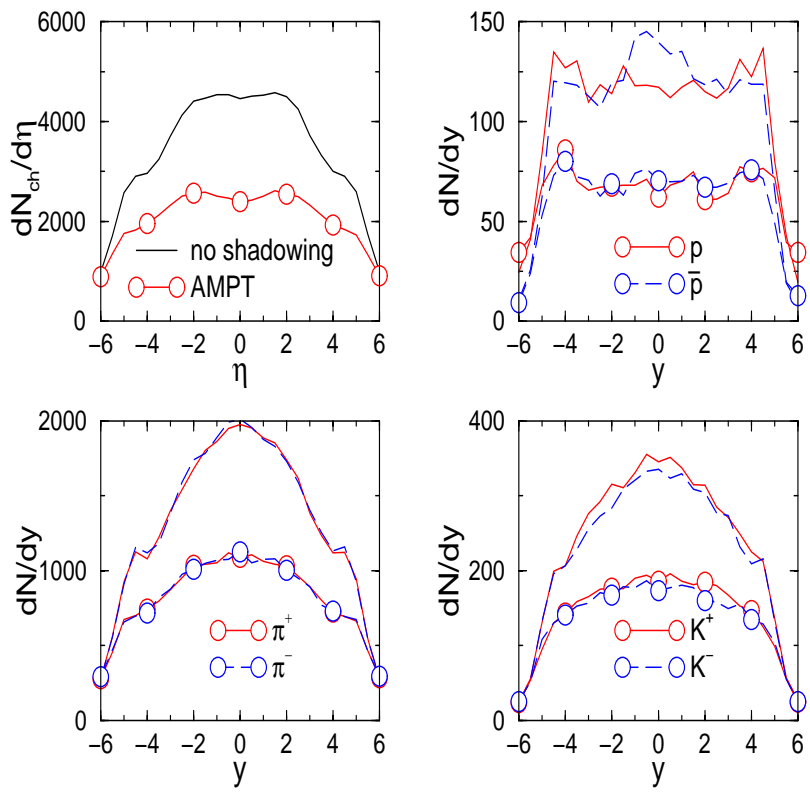

FIG. 1: (Color online) Rapidity distributions of charged hadrons (upper left panel), pions (lower left panel), kaons (lower right panel) as well as protons and antiprotons (upper right panel) for central ( $b \leq 3$ fm) $\mathrm{Pb}+\mathrm{Pb}$ collisions at $\sqrt{s_{N N}}=5.5 \mathrm{TeV}$ from the default AMPT model with (lines with circles) and without (lines without symbols) nuclear shadowing.

symbols) nuclear shadowing of nucleon parton structure functions are considered. Compared to those at RHIC, rapidity distributions at LHC are significantly wider and higher. At midrapidity, the distributions without shadowing are higher than corresponding ones with shadowing by about $80 \%$. The highest value at mid-rapidity is about 4500 , well within the LHC detector limit of 7000 particles per unit rapidity. The midrapidity density with nuclear shadowing is about 2500 , more than a factor of three higher than that at RHIC. It is also higher than the logarithmic extrapolation from lower energy data but lower than the saturation model prediction of about 3500 [53]. The charged hadron pseudorapidity distribution shows a clear plateau structure near midrapidity which is very different from predictions from saturation models $[54,55]$. The proton and antiproton rapidity distributions are close to each other and are almost flat. This is different from the proton and antiproton distributions at RHIC where protons are seen to dominate at large rapidities.

Most produced particles at LHC are from hard processes that are dependent of nucleon parton distribution functions. Since the parton distribution functions used in the present HIJING model is the old Duke-Owens set 1 [56] which gives far fewer partons at small- $x$ than more recent MRSA [57] and 4-flavor CTEQ6M [58] parameterizations, we might expect it to under-predict appreciably the particle multiplicity at LHC. However, saturation of gluons either in the initial nuclei or in the final state is expected to reduce the difference between predicted particle multiplicities from different parton structure functions. 


\section{B. Transverse momentum spectra}

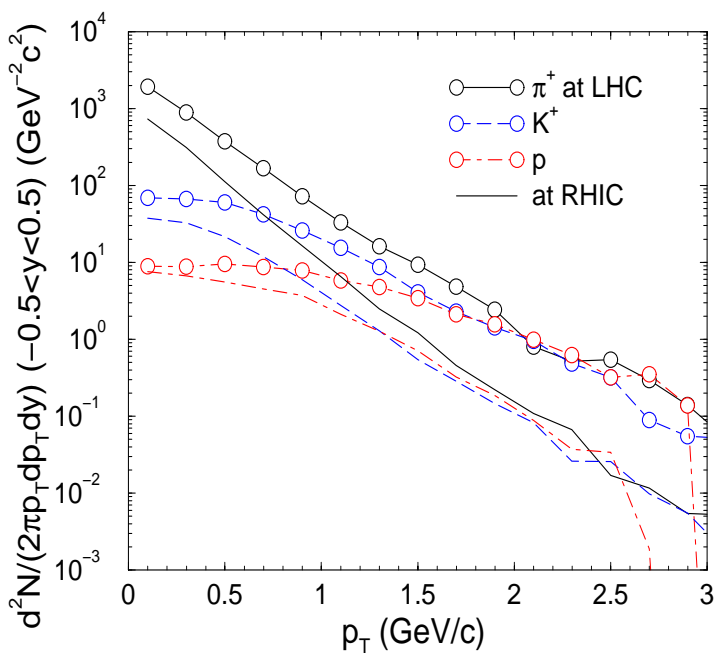

FIG. 2: (Color online) Transverse momentum spectra of pions (solid line), kaons (dashed line), and protons (dash-dotted line) from the default AMPT model for central $(b \leq 3 \mathrm{fm}) \mathrm{Pb}+\mathrm{Pb}$ collisions at $\sqrt{s_{N N}}=5.5 \mathrm{GeV}$ (lines with circles) and for central $(b \leq 3 \mathrm{fm}$ ) $\mathrm{Au}+\mathrm{Au}$ collisions at $\sqrt{s_{N N}}=200 \mathrm{GeV}$ (lines without symbols).

Using the default AMPT model, we have also studied the transverse momentum spectra of various hadrons in central $(b \leq 3 \mathrm{fm}) \mathrm{Pb}+\mathrm{Pb}$ collisions at $\sqrt{s_{N N}}=5.5 \mathrm{TeV}$ at LHC. As shown in Fig. 2 by lines with circles, their inverse slope parameters, particularly for kaons (dashed line) and protons (dashdotted line) with transverse momenta below $0.5 \mathrm{GeV} / c$ and 1 $\mathrm{GeV} / c$, respectively, are larger than those at RHIC which are shown by lines without symbols. The increased inverse slope parameters at LHC is due to a larger transverse flow as a result of stronger final-state rescattering. Similar to that observed at RHIC, the proton spectrum is below that of pions at low transverse momenta, but they become comparable at about 2 $\mathrm{GeV} / c$.

\section{Elliptic flow}

The elliptic flow in heavy ion collisions measures the asymmetry of particle momentum distributions in the plane perpendicular to the beam direction. It is defined as one half of the second Fourier coefficient of the azimuthal angle distribution of particle transverse momentum and can be evaluated via

$$
v_{2}=\left\langle\frac{p_{x}^{2}-p_{y}^{2}}{p_{x}^{2}+p_{y}^{2}}\right\rangle \text {. }
$$

In the above, the $x$-axis is along the impact parameter in the transverse plane of each event while the $y$-axis points out of the reaction plane, and the average is taken over considered particles. It results from initial spatial asymmetry in non-central collisions [59,60]. Theoretical studies have shown that the elliptic flow is sensitive to the properties of the hot dense matter formed during the initial stage of heavy ion collisions $[6,11,61-65]$. In particular, it was shown in Refs. $[64,65]$ via the parton cascade model that the parton elliptic flow increases with the magnitude of parton scattering cross section. With a cross section of $10 \mathrm{mb}$ for partons in the partonic cascade, the AMPT model with string melting has satisfactorily reproduced the transverse momentum [41], rapidity [48], and flavor [51, 52] dependence of measured elliptic flow at RHIC. In this section, predicted elliptic flow in heavy ion collisions at LHC from the AMPT model with string melting is shown for both light and heavy quarks as well as resulting hadrons.

\section{Light quarks and hadrons}

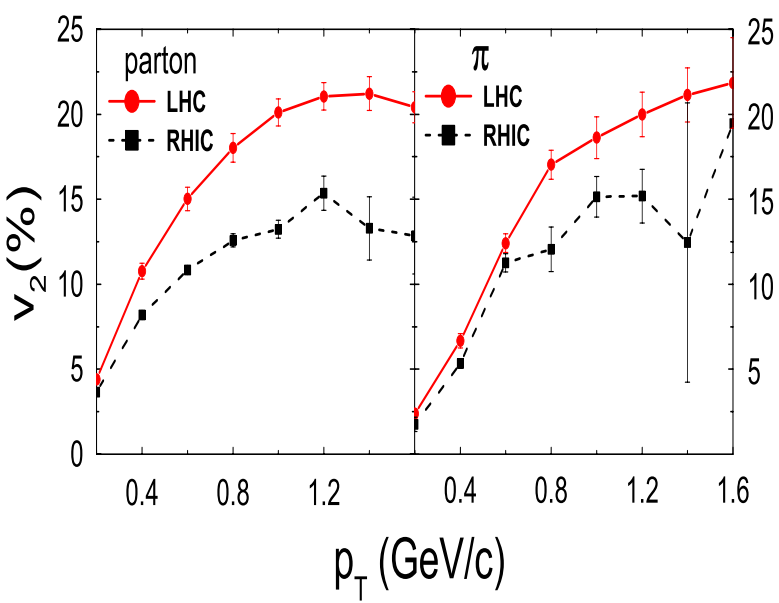

FIG. 3: (Color online) Elliptic flows of light quarks (left panel) and pions (right panel) in $\mathrm{Pb}+\mathrm{Pb}$ collisions at $\sqrt{s_{N N}}=5.5 \mathrm{TeV}$ and impact parameter $b=8 \mathrm{fm}$ from the AMPT model with string melting and parton cross section of $10 \mathrm{mb}$.

In Fig. 3, we show by solid lines the transverse momentum dependence of the elliptic flows of light quarks (left panel) and pions (right panel) obtained from the AMPT model using a parton cross section of $10 \mathrm{mb}$ for $\mathrm{Pb}+\mathrm{Pb}$ collisions at centerof-mass energy $\sqrt{s_{N N}}=5.5 \mathrm{TeV}$ and impact parameter $b=8$ fm. Also shown by dashed lines are parton and pion elliptic flows at RHIC for Au+Au collisions at $\sqrt{s_{N N}}=200 \mathrm{GeV}$ and same impact parameter. It is seen that both light quark and pion elliptic flows at LHC are larger than corresponding ones at RHIC.

In obtaining above results, we have used same parton scattering cross section in the partonic matter produced at LHC as that at RHIC. The parton scattering cross section used in the AMPT model is an effective one that depends on the properties of the partonic matter formed in a collision. The large parton cross section needed to reproduce measured elliptic flow at RHIC indicates that the partonic matter produced at RHIC is strongly interacting, consistent with the conclusion based 
on the hydrodynamic model [11-13] as well as the prediction from the lattice QCD study. Since the energy density or temperature of the partonic matter produced at LHC is expected to be much higher than those at RHIC, the partonic matter might become less strongly interacting, leading to a smaller effective parton scattering cross section and thus a smaller elliptic flow. Studying the elliptic flow at LHC can therefore provide important information on the properties of the partonic matter at high temperature.

\section{Heavy quarks and mesons}

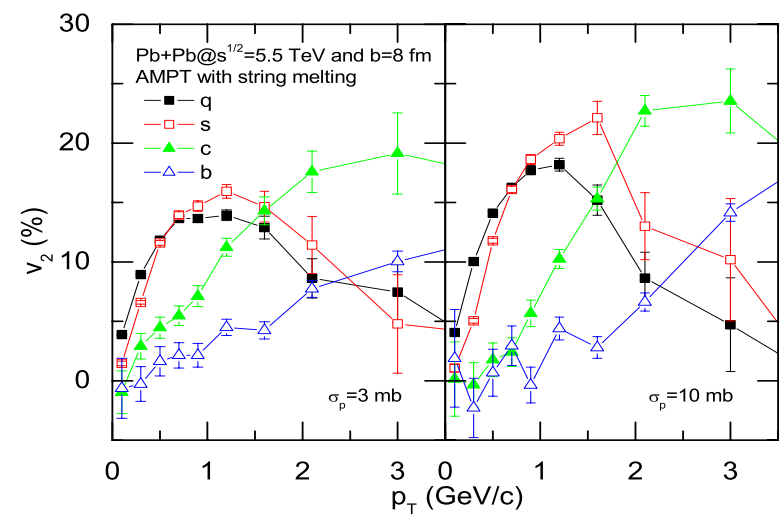

FIG. 4: (Color online) Elliptic flows of light, strange, and heavy quarks in $\mathrm{Pb}+\mathrm{Pb}$ collisions at $\sqrt{s_{N N}}=5.5 \mathrm{TeV}$ and $b=8 \mathrm{fm}$ from the AMPT model with string melting and parton scattering cross sections of 3 (left panel) and 10 (right panel) mb.

For heavy charm and bottom quarks, their elliptic flows from the AMPT model with string melting for $\mathrm{Pb}+\mathrm{Pb}$ collisions at $\sqrt{s_{N N}}=5.5 \mathrm{TeV}$ and $b=8 \mathrm{fm}$ are shown in Fig. 4 by filled and open triangles, respectively, for two parton scattering cross sections of 3 (left panel) and 10 (right panel) mb. Besides increasing with parton scattering cross section, quark elliptic flows display the expected mass ordering at low transverse momentum, i.e., the elliptic flow of charm quarks is larger than that of bottom quarks but smaller than that of light and strange quarks, which are shown by filled and open squares, respectively. The elliptic flows of heavy quarks further increase with their transverse momentum and become larger than those of light and strange quarks which peak at around 1-1.5 GeV/c. They are, however, expected to eventually decrease at high transverse momentum but are not shown in the figure due to low statistics.

From elliptic flows of heavy quarks, we can estimate those of heavy mesons using the quark coalescence or recombination model $[27,66]$. Since coalescence is predominately among quarks with similar velocity, the elliptic flow $v_{2, M}\left(p_{T}\right)$ at transverse momentum $p_{T}$ of heavy mesons consisting of one heavy and one light quark is approximately given by [27]

$$
v_{2, M}\left(p_{T}\right) \approx v_{2, Q}\left(\left(m_{Q} / m_{M}\right) p_{T}\right)+v_{2, q}\left(\left(m_{q} / m_{M}\right) p_{T}\right) .
$$

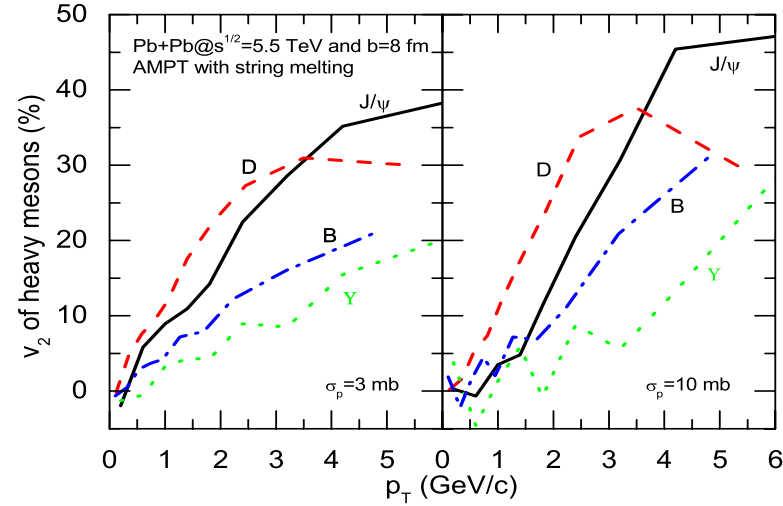

FIG. 5: (Color online) Elliptic flows of heavy mesons in $\mathrm{Pb}+\mathrm{Pb}$ collisions at $\sqrt{s_{N N}}=5.5 \mathrm{TeV}$ and $b=8 \mathrm{fm}$ from the quark coalescence model using the quark elliptic flows from the AMPT model with string melting and parton scattering cross sections of 3 (left panel) and 10 (right panel) $\mathrm{mb}$.

In the above, $v_{2, Q}$ and $v_{2, q}$ are elliptic flows of heavy and light quarks, respectively; while $m_{M}, m_{Q}$, and $m_{q}$ are, respectively, masses of heavy meson, heavy quark, and light quark.

Using $m_{q}=300 \mathrm{MeV}, m_{c}=1.5 \mathrm{GeV}$ and $m_{b}=4.8 \mathrm{GeV}$ together with the elliptic flows of quarks shown in Fig. 5, we have obtained the elliptic flows of heavy open charmed $(D)$ and bottomed (B) mesons based on Eq.(3). The results are shown in Fig. 5. Because of much larger heavy quark masses than those of light quarks, the elliptic flows of heavy mesons are close to those of heavy quarks, particularly for bottomed mesons. Eq.(3) can be generalized to heavy mesons with hidden charm or bottom, i.e., quarkonia $J / \psi$ and $\Upsilon$ consisting of a heavy quark and its antiquark. Their elliptic flows at $p_{T}$ are then twice that of their constituent heavy quarks at $p_{T} / 2$ and are also shown in Fig. 5.

The elliptic flows of heavy mesons have already been studied at RHIC via measurement of their decay electrons [67, 68]. The observed large value in $\mathrm{Au}+\mathrm{Au}$ collisions at $\sqrt{s_{N N}}=200$ $\mathrm{GeV}$ is consistent with large elliptic flows of heavy quarks, particular that of charmed quarks as shown in Refs.[51, 69] based on the quark coalescence model. Without heavy quark elliptic flow, resulting heavy meson elliptic flow would be much smaller as shown in Ref.[70]. Studying heavy meson elliptic flow at LHC is thus very useful for understanding the dynamics of heavy quarks in partonic matter.

\section{Two-pion interferometry}

Particle interferometry based on the Hanbury-Brown Twiss (HBT) effect can provide information on not only the spatial extent of the emission source but also its expansion velocity and emission duration [3, 4, 71-73]. In particular, the long emission time as a result of phase transition from the quarkgluon plasma to the hadronic matter in relativistic heavy ion collisions is expected to lead to an emission source which has a much larger radius in the direction of the total transverse 
momentum of detected two particles $\left(R_{\text {out }}\right)$ than that perpendicular to both this direction and the beam direction $\left(R_{\text {side }}\right)$ $[71,74,75]$. Although the extracted ratio $R_{\text {out }} / R_{\text {side }}$ from a Gaussian fit to the measured two-pion correlation function in $\mathrm{Au}+\mathrm{Au}$ collisions at $\sqrt{s_{N N}}=130 \mathrm{GeV}$ is close to one [7678], the source function extracted from the imaging method seems to show a longer tail in the out direction compared to other directions [79]. The small value of $R_{\text {out }} / R_{\text {side }}$ has been attributed to strong space-time and momentum correlations in the emission source [80]. Since the quark-gluon plasma produced at LHC will be at even higher temperature, the emission source is expected to also have a large radius in the out direction.

\section{Two-particle correlation function}

The single-particle emission function $S(x, \mathbf{p})$ in the AMPT model is given by the space-time coordinate $x$ and momentum $\mathbf{p}$ of particles at freeze-out, i.e., at their last interactions. In terms of the emission function, the HBT correlation function for two identical particles of momenta $\mathbf{p}_{1}$ and $\mathbf{p}_{2}$ in the absence of final-state interactions, such as the Coulomb interaction, is then given by $[3,81]$ :

$$
\begin{aligned}
& C_{2}(\mathbf{Q}, \mathbf{K}) \\
& \approx 1+\frac{\int d^{4} x_{1} d^{4} x_{2} S\left(x_{1}, \mathbf{p}_{1}\right) S\left(x_{2}, \mathbf{p}_{2}\right) \cos \left[Q \cdot\left(x_{1}-x_{2}\right)\right]}{\int d^{4} x_{1} S\left(x_{1}, \mathbf{p}_{1}\right) \int d^{4} x_{2} S\left(x_{2}, \mathbf{p}_{2}\right)}
\end{aligned}
$$

where $\mathbf{K}=\left(\mathbf{p}_{1}+\mathbf{p}_{2}\right) / 2$ and $Q=\left(\mathbf{p}_{1}-\mathbf{p}_{2}, E_{1}-E_{2}\right)$. The threedimensional correlation function in $\mathbf{Q}$ is usually shown as a function of the invariant relative momentum $\left(Q_{\mathrm{inv}}=\sqrt{-Q^{2}}\right)$ or as a function of the projection of the relative momentum $\mathbf{Q}$ in the "out-side-long" (osl) system [72, 73], defined by the beam direction $\left(Q_{\text {long }}\right)$, the direction along the total momentum of the two particles in the transverse plane $\left(Q_{\text {out }}\right)$, and the direction orthogonal to above two directions $\left(Q_{\text {side }}\right)$.

Using the emission function obtained from the AMPT model with string melting and a parton scattering cross section of $10 \mathrm{mb}$ for central $(b=0 \mathrm{fm}) \mathrm{Pb}+\mathrm{Pb}$ collisions at $\sqrt{s_{N N}}=$ 5.5 TeV, we have evaluated the correlation function $C_{2}(\mathbf{Q}, \mathbf{K})$ in the longitudinally comoving frame using the program Correlation After Burner [82] that takes into account final-state strong and Coulomb interactions between two charged pions. In Fig. 6, we show one-dimensional projections of calculated correlation functions including final-state Coulomb interactions for midrapidity $(-0.5<y<0.5)$ charged pions with transverse momentum $125<p_{\mathrm{T}}<225 \mathrm{MeV} / c$. For comparison, correlation functions for charged pions in the same momentum range for central $\mathrm{Au}+\mathrm{Au}$ collisions at $\sqrt{s_{N N}}=200$ $\mathrm{GeV}$ at RHIC are also given in Fig. 6 by dashed lines, which have been shown to reproduce reasonably measured ones [43]. It is seen that the correlation functions have smaller values at LHC than at RHIC.

\section{2. $\quad$ Source radii}

The size of the emission source can be determined from the emission function via the curvature of the correlation function

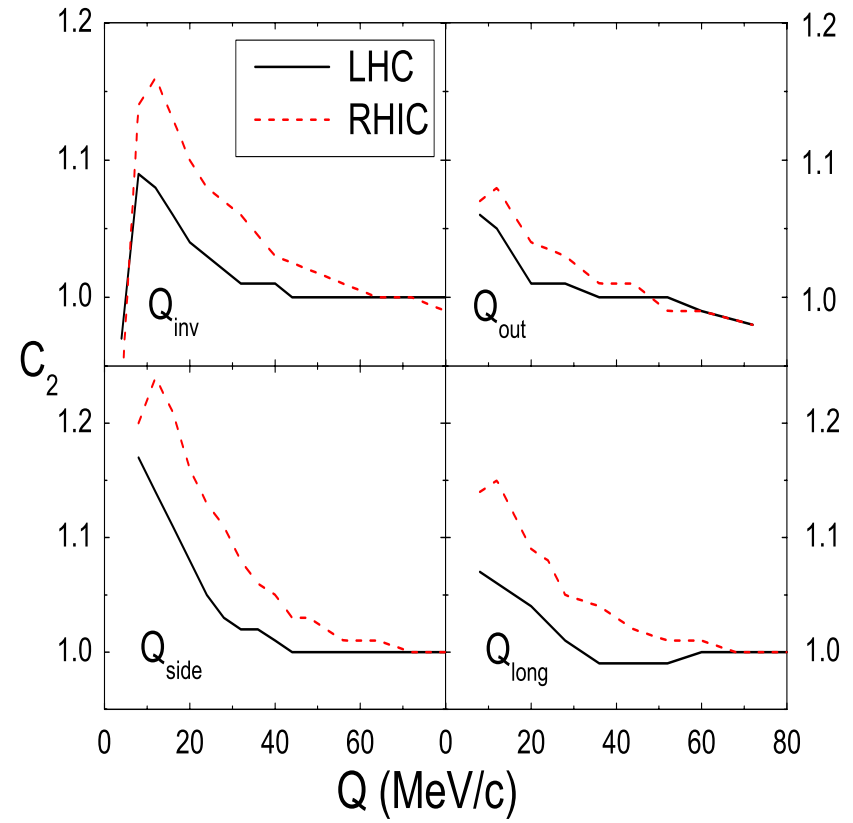

FIG. 6: (Color online) Correlation functions for midrapidity charged pions with $125<p_{\mathrm{T}}<225 \mathrm{MeV} / c$ from the AMPT model with string melting and parton cross section of $10 \mathrm{mb}$ for central $(b=0$ fm) $\mathrm{Pb}+\mathrm{Pb}$ collisions at $\sqrt{s_{N N}}=5.5 \mathrm{TeV}$ (solid lines) and $\mathrm{Au}+\mathrm{Au}$ collisions at $\sqrt{s_{N N}}=200 \mathrm{GeV}$ (dashed lines).

at $\mathbf{Q}=0$ :

$$
\begin{aligned}
& R_{i j}(K)^{2}=-\left.\frac{1}{2} \frac{\partial^{2} C_{2}(\mathbf{Q}, \mathbf{K})}{\partial Q_{i} \partial Q_{j}}\right|_{\mathbf{Q}=0} \\
& =D_{x_{i}, x_{j}}(K)-D_{x_{i}, \beta_{j} t}(K)-D_{\beta_{i} t, x_{j}}(K)+D_{\beta_{i} t, \beta_{j} t}(K),
\end{aligned}
$$

where $\beta=\mathbf{K} / K_{0}$ with $K_{0}$ being the average energy of the two particles. In the second line of Eq.(5), we have the variance $D_{x, y}=\langle x \cdot y\rangle-\langle x\rangle\langle y\rangle$, with $\langle x\rangle$ denoting the average value of $x$. If the emission source is Gaussian in space-time, then for central heavy ion collisions considered here these radius parameters would be the same as those obtained from a Gaussian fit to the correlation function.

The source radii evaluated using Eq.(5) are shown in Fig. 7 by solid lines for central $\mathrm{Pb}+\mathrm{Pb}$ collisions at $\sqrt{s_{N N}}=5.5 \mathrm{TeV}$ at LHC. Compared to those in $\mathrm{Au}+\mathrm{Au}$ collisions at $\sqrt{s_{N N}}=$ $200 \mathrm{GeV}$ at RHIC, shown by dashed lines, radii of the emission source at LHC are larger. The ratio $R_{\text {out }} / R_{\text {side }}$ shown in the lower right panel is, however, smaller at LHC than at RHIC. In both cases, the ratio is larger than one and would have been even larger if not for the positive $x_{\text {out }}-t$ correlation, where $x_{\text {out }}$ is the projection of the particle position at freezeout in the out direction of the osl system. This is shown in the right panel of Fig. 8 together with the $x_{\text {out }}-x_{\text {side }}$ distribution (left panel), where $x_{\text {side }}$ being the projection in the side direction of the $o s l$ system. Also shown in Fig. 8 by filled circles is the correlation between the average value $\left\langle x_{\text {out }}\right\rangle$ with the freeze-out time $t$. As at RHIC, the emission source at LHC is non-Gaussian and shifted in the direction of pion transverse momentum. For pions included in generating Fig. 8, i.e., with transverse momentum $125<p_{\mathrm{T}}<225 \mathrm{MeV} / c, D_{x_{\text {out }}, \beta_{\perp} t}$ $\left(=D_{\beta_{\perp} t, x_{\text {out }}}\right.$, has a value of about $233 \mathrm{fm}^{2}$ and is appreciable 


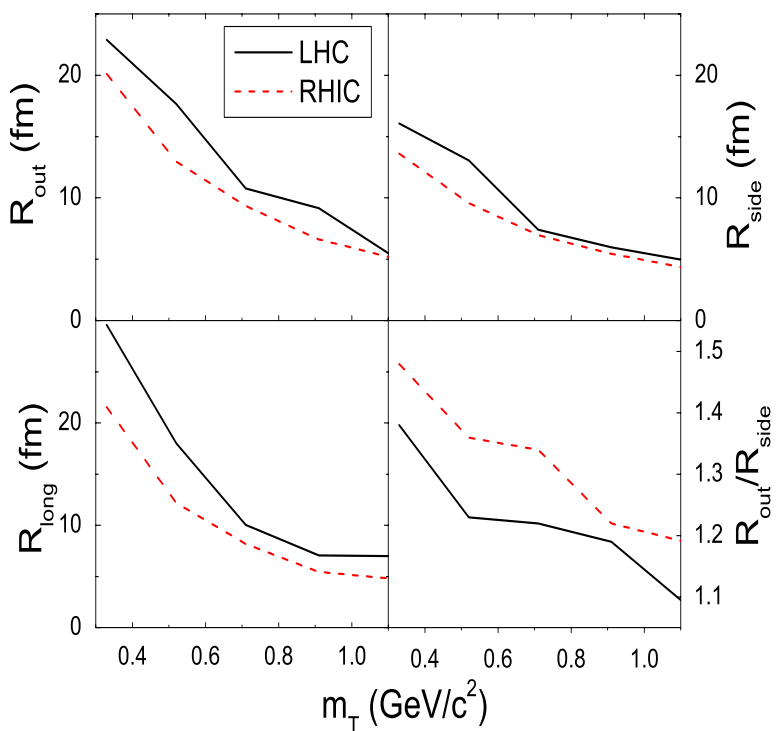

FIG. 7: (Color online) Source radii from the emission function for midrapidity pions as functions of pion transverse mass $m_{\mathrm{T}}$ from the AMPT model with string melting and parton cross section of $10 \mathrm{mb}$ for central $(b=0 \mathrm{fm}) \mathrm{Pb}+\mathrm{Pb}$ collisions at $\sqrt{s_{N N}}=5.5 \mathrm{TeV}$ at LHC (solid lines) and $\mathrm{Au}+\mathrm{Au}$ collisions at $\sqrt{s_{N N}}=200 \mathrm{GeV}$ at RHIC (dashed lines).

compared to about 356 and $756 \mathrm{fm}^{2}$, respectively, for $D_{x_{\text {out }}, x_{\text {out }}}$ and $D_{\beta_{t} t, \beta_{\perp} t}$ in the first and last terms in Eq.(5). This makes it difficult to extract information about the duration of emission from the ratio $R_{\text {out }} / R_{\text {side }}$ as already demonstrated in studies based on the AMPT model for heavy ion collisions at RHIC [43] and the RQMD model for heavy ion collisions at SPS $[83,84]$.

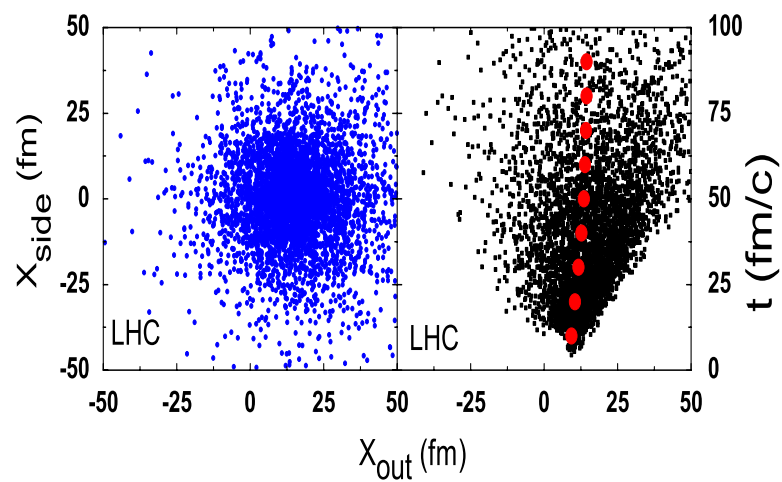

FIG. 8: (Color online) $x_{\text {out }}-x_{\text {side }}$ (left panel) and $x_{\text {out }}-t$ (right panel) distributions at freeze-out for midrapidity charged pions with $125<p_{\mathrm{T}}<225 \mathrm{MeV} / c$ from the AMPT model with string melting and parton cross section of $10 \mathrm{mb}$. The line with filled circles represents $\left\langle x_{\text {out }}\right\rangle$ as a function of $t$.

\section{Pair separation function}

The non-Gaussian feature of the emission source can be more directly inferred from the correlation function by using the imaging method $[85,86]$. In this method, the pair separation function $S(\mathbf{r})$, which gives the probability distribution of emitting a pair of particles with separation $\mathbf{r}$ in their centerof-mass frame, is obtained from measured two-particle correlation function by the inversion of the Koonin-Pratt equation [87]:

$$
C(\mathbf{q})-1=\int d^{3} \mathbf{r}\left[\left|\phi_{\mathbf{q}}^{(-)}(\mathbf{r})\right|^{2}-1\right] S(\mathbf{r}),
$$

where $\phi_{\mathbf{q}}^{(-)}$is the relative wave function of the two particles with relative momentum $\mathbf{q}$ in their center-of-mass frame. For heavy-ion collisions at RHIC, extracted pair separation function from measured two-pion correlation functions at midrapidity in central $\mathrm{Au}+\mathrm{Au}$ collisions at $\sqrt{s_{N N}}=200 \mathrm{GeV}$ by the PHENIX collaboration [88] indicates that the pion emission source has a long tail beyond the Gaussian shape as shown by filled triangles in Fig. 9.

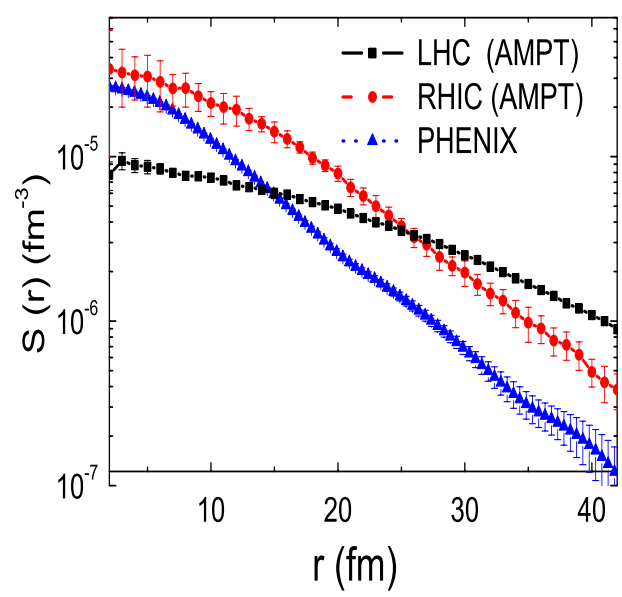

FIG. 9: (Color online) Separation functions for midrapidity pion pairs with average transverse momentum $0.20<K_{T}<0.36 \mathrm{GeV} / c$ from the AMPT model with string melting and parton cross section of $10 \mathrm{mb}$ for central $(b=0 \mathrm{fm}) \mathrm{Pb}+\mathrm{Pb}$ collisions at $\sqrt{s_{N N}}=5.5 \mathrm{TeV}$ at LHC (filled squares) and Au+Au collisions at $\sqrt{s_{N N}}=200 \mathrm{GeV}$ at RHIC (filled circles). Filled triangles are from the imaging method using the two-pion correlation functions measured by the PHENIX collaboration [88].

In the AMPT model, the pair separation function $S(\mathbf{r})$ can be straightforwardly evaluated from the positions and momenta of particles at freeze-out. The predicted separation functions for midrapidity pion pairs with average transverse momentum $0.20<K_{T}<0.36 \mathrm{GeV} / c$ from the AMPT model with string melting and parton cross section of $10 \mathrm{mb}$ are shown in Fig. 9 for central $(b=0 \mathrm{fm}) \mathrm{Pb}+\mathrm{Pb}$ collisions at $\sqrt{s_{N N}}=5.5 \mathrm{TeV}$ at LHC (filled squares) and $\mathrm{Au}+\mathrm{Au}$ collisions at $\sqrt{s_{N N}}=200 \mathrm{GeV}$ at RHIC (filled circles). They are normalized to unity when integrated over the separation, i.e., $\int d^{3} \mathbf{r} S(\mathbf{r})=1$. Compared with the pion pair separation 
function extracted from measured correlation functions by the imaging method (filled triangles), which has a normalization of about 0.4 as not all emitted pions contribute to the extracted emission source, the pair separation function from the AMPT model has a somewhat longer tail than the empirical one. More detailed study of the pair separation function in terms of its projection to the osl system using the Cartesian harmonics [89] shows that the projected pair separation functions from the AMPT model in the out and long directions are consistent with those extracted from the imaging method while that in the side direction has a longer tail than the empirical one. The reason for the latter is presently not understood. The predicted pair separation function from the AMPT model has an even longer tail for heavy ion collisions at LHC than at RHIC as shown in Fig. 9. The predicted appreciable difference in the pion pair separation functions at LHC and RHIC should be testable experimentally.

\section{SUMMARY}

To predict what one might observe in $\mathrm{Pb}+\mathrm{Pb}$ collisions at $\sqrt{s_{N N}}=5.5 \mathrm{TeV}$ that will soon be available at LHC, we have used a multiphase AMPT transport model that includes scattering in both initial partonic and final hadronic matters and the transition between these two phases of matter. Using a large parton scattering cross section of $10 \mathrm{mb}$, which is needed to describe observations in $\mathrm{Au}+\mathrm{Au}$ collisions at $\sqrt{s_{N N}}=200$ $\mathrm{GeV}$ at RHIC, we have studied the rapidity distributions and transverse momentum spectra of various hadrons, the elliptic flows of both light and heavy quarks as well as resulting hadrons from coalescence of quarks and antiquarks, and the two-pion correlation functions as well as the emission source function.

For rapidity distributions, the total charge multiplicity in central $\mathrm{Pb}+\mathrm{Pb}$ collisions at $\mathrm{LHC}$ is almost a factor of three larger than in central $\mathrm{Au}+\mathrm{Au}$ collisions at RHIC. This value may, however, subject to large uncertainty due to the nucleon structure functions at small $x$ that is used in the model. Compared to those at RHIC, hadron transverse momentum spectra show even larger effective inverse slope parameters as a result of stronger collective transverse flow.

For non-central collisions, the elliptic flows of quarks at LHC are stronger than those at RHIC. Although their values at low transverse momentum decrease with the mass of quark, they reach similar peak values at higher transverse momentum. Using the quark coalescence model, the elliptic flows of hadrons including those consisting of heavy quarks can be related to those of quarks and thus provide the possibility to study the partonic dynamics in the collisions.

From the emission function of pions in the AMPT model, two-pion correlation functions have been evaluated and are found to be smaller than those at RHIC. As at RHIC, the emission source is non-Gaussian in space and time. It shifts significantly to the direction along the pion transverse momentum and also has a strong correlation between this displacement and the emission time, leading to a radius in the direction along the pion momentum that is only somewhat larger than the radius in the direction that is perpendicular to the pion momentum and the beam direction. All radii of the emission source at LHC are, however, larger than those at RHIC. The emission function from the AMPT model also allows one to calculate the pair separation function of the source and to compare with the empirical one extracted from measured correlation function by the imaging method.

The above results are obtained with an effective parton scattering cross section of $10 \mathrm{mb}$. With a smaller parton cross section, which may be the case as the energy density achieved at LHC is expected to be much larger than that at RHIC, all particles would have smaller effective inverse slope parameters in their transverse momentum spectra, smaller elliptic flows, and larger two-particle correlations or smaller radii for the emission source than what have been shown here. Comparing heavy ion collisions at LHC against those at RHIC is thus expected to add greatly to our understanding of the properties of produced partonic matter.

\section{Acknowledgments}

We thank Paul Chung for helpful communications on the pair separation function of emission source. This work was supported in part by the US National Science Foundation under Grant No. PHY-0457265 and the Welch Foundation under Grant No. A-1358 (CMK), the National Natural Science Foundation of China under Grant Nos. 10405011 (BWZ) as well as 10575071 and 10675082 , MOE of China under project NCET-05-0392, Shanghai Rising-Star Program under Grant No. 06QA14024, and the SRF for ROCS, SEM of China (LWC).
[1] E. Shuryak, Phys. Lett. B 78, 150 (1978).

[2] J. Rafelski and B. Müller, Phys. Lett. B 101, 111 (1982).

[3] S. Pratt, Phys. Rev. Lett. 53, 1219 (1984); Phys. Rev. D 33, 1314 (1986).

[4] G. F. Bertsch, Nucl. Phys. A 498, 173c (1989).

[5] T. Matsui and H. Satz, Phys. Lett. B 178, 416 (1986).

[6] J.Y. Ollitrault, Phys. Rev. D 46, 229 (1992).

[7] X. N. Wang and M. Gyulassy, Phys. Rev. Lett. 68, 1480 (1992).

[8] S. Voloshin, Nucl. Phys. A 715, 379 (2003).

[9] P. Braun-Munzinger, D. Majestra, K. Redlich, and J. Stachel,
Phys. Lett. B 518, 41 (2001).

[10] G. Torrieri and J. Rafelski, J. Phys. G 30, 557 (2004).

[11] D. Teaney, J. Lauret, and E. V. Shuryak, Phys. Rev. Lett. 86 4783 (2001); Nucl. Phys. A 698, 479 (2002).

[12] P. Huovinen, P. F. Kolb, and U. Heinz, Nucl. Phys. A 698, 475 (2002).

[13] T. Hirano and K. Tsuda, Phys. Rev C 66, 054905 (2002).

[14] B. Zhang, C. M. Ko, B. A. Li, and Z. W. Lin, Phys. Rev. C 61, 067901 (2000).

[15] Z. W. Lin, S. Pal, C. M. Ko, B. A. Li, and B. Zhang, Phys. Rev. 
C 64, 011902 (2001); Nucl. Phys. A 698, 375 (2002).

[16] Z. W. Lin, C. M. Ko, B. A. Li, B. Zhang, and S. Pal, Phys. Rev. C 72, 064901 (2005).

[17] D. Molnar and M. Gyulassy, Nucl. Phys. A 697, 495 (2002) [Erratum-ibid. A 703, 893 (2002)].

[18] W. Cassing and E. L. Bratkovskaya, Phys. Rep. 308, 65 (1999).

[19] S. Bass et al., Prog. Part. Nucl. Phys. 41, 225 (1998).

[20] B. H. Sa, X. Cai, Z. D. Su, A. Tai, and D. M. Zhou, Phys. Rev. C 66, 044902 (2002).

[21] D. M. Zhou, X. M. Li, B. G. Dong, and B. H. Sa, Phys. Lett. B 638, 461 (2006).

[22] L. Bravina, K. Tywoniuk, E. Zabrodin, G. Burau, J. Bleibel, C. Fuchs, and A. Faessler, Phys. Lett. B 631, 109 (2005).

[23] Z. Xu and C. Greiner, Phys. Rev. C 71, 064901 (2005).

[24] R. Hwa and C. B. Yang, Phys. Rev. C 67, 034902 (2003).

[25] V. Greco, C. M. Ko, and P. Lévai, Phys. Rev. Lett. 90, 202302 (2003); Phys. Rev. C 68, 034904 (2003).

[26] R. J. Fries, B. Müller, C. Nonaka, and S. A. Bass, Phys. Rev. Lett. 90, 202303 (2003); Phys. Rev. C 68, 044902 (2003).

[27] Z. W. Lin and D. Molnar, Phys. Rev. C 68, 044901 (2003).

[28] M. Gyulassy and X. N. Wang, Nucl. Phys. B 420, 583 (1994).

[29] M. Gyulassy, P. Levai, and I. Vitev, Phys. Rev. Lett. 85, 5535 (2001).

[30] J. Adams et al., [STAR Collaboration], Nucl. Phys. A 757, 102 (2005).

[31] B. Zhang, C. M. Ko, B. A. Li, Z. W. Lin, and B. H. Sa, Phys. Rev. C 62, 054905 (2000).

[32] B. Zhang, C. M. Ko, B. A. Li, Z. W. Lin, and S. Pal, Phys. Rev. C 65, 054909 (2002)

[33] C. M. Ko, Z. W. Lin, and S. Pal, Heavy Ion Phys. 17, 219 (2003).

[34] S. Pal. C. M. Ko, and Z. W. Lin, Nucl. Phys. A 730, 143 (2004).

[35] X. N. Wang and M. Gyulassy, Phys. Rev. D 44, 3501 (1991).

[36] B. Zhang, Comput. Phys. Commun. 109, 193 (1998).

[37] T. Sjostrand, Comput. Phys. Commun. 82, 74 (1994);

[38] B. A. Li and C. M. Ko, Phys. Rev. C 52, 2037 (1995),

[39] B.A. Li, A.T. Sustich, B. Zhang, and C.M. Ko, Int. Jour. Phys. E 10, 267-352 (2001).

[40] D. Kharzeev and M. Nardi, Phys. Lett. B 507, 121 (2001).

[41] Z. W. Lin and C. M. Ko, Phys. Rev. C 65, 034904 (2002).

[42] L. W. Chen, C. M. Ko, and Z. W. Lin, Phys. Rev. C 69, 031901 (2004)

[43] Z. W. Lin, C. M. Ko, and S. Pal, Phys. Rev. Lett. 89, 152301 (2002).

[44] K. H. Ackermann et al., [STAR Collaboration], Phys. Rev. Lett. 86, 402 (2001)

[45] J. Adams et al., [STAR Collaboration], Phys. Rev. lett. 92, 062301 (2004)

[46] C. Adler et al., [STAR Collaboration], Phys. Rev. Lett. 87, 082301 (2001).

[47] Z. W. Lin and C. M. Ko, J. Phys. G 30, S263 (2004).

[48] L. W. Chen, V. Greco, C. M. Ko, and P. F. Kolb, Phys. Lett. B 605, 95 (2005).

[49] L. W. Chen and C. M. Ko, Phys. Lett. B 634, 205 (2006).

[50] L. W. Chen and C. M. Ko, Phys. Rev. C 73, 014906 (2006).

[51] B. Zhang, L. W. Chen, and C. M. Ko, Phys. Rev. C 72, 024906 (2005).

[52] L. W. Chen and C. M. Ko, Phys. Rev. C, 73, 044903 (2006).

[53] Y. Schutz, J. Phys. G 30, S903 (2004).

[54] N. Armesto, C. A. Salgado, and U. A. Wiedemann, Phys. Rev. Lett. 94, 022002 (2005).
[55] D. Kharzeev, E. Levin, and M. Nardi, Nucl. Phys. A 747, 609 (2005).

[56] D. W. Duke and J. F. Owens, Phys. Rev. D 30, 49 (1984).

[57] A. D. Martin, W. J. Stirling, and R. G. Roberts, Phys. Rev. D 50, 6734 (1994).

[58] S. Kretzer, H. L. Lai, F. I. Olness, and W. K. Tung, Phys. Rev. D 69, 114005 (2004).

[59] J. Barrette et al. [E877 Collaboration], Phys. Rev. Lett. 73, 2532 (1994).

[60] H. Appelshauser et al. [NA49 Collaboration], Phys. Rev. Lett. 80, 4136 (1998).

[61] H. Sorge, Phys. Rev. Lett. 78, 2309 (1997); Phys. Rev. Lett. 82 2048 (1999).

[62] P. Danielewicz, R. A. Lacey, P. B. Gossiaux, C. Pinkenburg, P. Chung, J. M. Alexander, and R. L. McGrath, Phys. Rev. Lett. 81, 2438 (1998).

[63] Y. Zheng, C. M. Ko, B. A. Li, and B. Zhang, Phys. Rev. Lett. 83, 2534 (1999).

[64] B. Zhang, M. Gyulassy, and C. M. Ko, Phys. Lett. B 455, 45 (1999).

[65] D. Molnar and M. Gyulassy, Nucl. Phys. A 698, 379 (2002);

[66] V. Greco, C. M. Ko, and R. Rapp, Phys. Lett. B 595, 202 (2004).

[67] PHENIX Collaboration, S.S. Adler et al., Phys. Rev. Lett. 96 032301 (2006).

[68] J. Bielcik and STAR Collaboration, Nucl. Phys. A 774, 697 (2006).

[69] H. van Hees, V. Greco, and R. Rapp, Phys. Rev. C 73, 034913 (2006).

[70] V. Greco and C. M. Ko, Phys. Lett. B 595, 202 (2004).

[71] D. H. Rischke and M. Gyulassy, Nucl. Phys. A 608, 479 (1996).

[72] G. Bertsch, M. Gong, and M. Tohyama, Phys. Rev. C 37, 1896 (1988).

[73] S. Pratt, T. Csörgö, and J. Zimanyi, Phys. Rev. C 42, 2646 (1990).

[74] S. Soff, S. A. Bass, and A. Dumitru, Phys. Rev. Lett. 86, 3981 (2001).

[75] S. Soff, S. A. Bass, D. H. Hardtke, and S. Y. Panitkin, Phys. Rev. Lett. 88, 072301 (2002).

[76] C. Adler et al., [STAR Collaboration], Phys. Rev. Lett. 87, 082301 (2001).

[77] S. C. Johnson, [PHENIX Collaboration], Nucl. Phys. A 698, 603 (2002)

[78] K. Adcox et al., [PHENIX Collaboration], Phys. Rev. Lett. 88 192302 (2002).

[79] P. Chung and R. Lacey, paper in this volume.

[80] B. Tomasik and U. W. Heinz, nucl-th/9805016.

[81] U. A. Wiedemann and U. W. Heinz, Phys. Rept. 319, 145 (1999) .

[82] S. Pratt et al., Nucl. Phys. A 566, 103c (1994).

[83] J. P. Sullivan et al., Phys. Rev. Lett. 70, 3000 (1993).

[84] D. E. Fields, J. P. Sullivan, J. Simon-Gillo, H. van Hecke, B. V. Jacak, and N. Xu, Phys. Rev. C 52, 986 (1995).

[85] D. A. Brown and P. Danielewicz, Phys. Lett. B 398, 252 (1997); Phys. Rev. C 57, 2474 (1998); ibid 64, 014902 (2001).

[86] S. Y. Panitkin and D. A. Brown, Phys. Rev. C 61, 021901 (2000).

[87] S. E. Koonin, Phys. Lett. B 70, 43 (1977).

[88] S.S. Adler et al., PHENIX Collaboration, nucl-ex/0605032.

[89] P. Danielewics and S. Pratt, Phys. Lett. B 618, 60 (2005). 